\title{
Re-signaling the Paris Line 1: from driver-based to driverless operation
}

\author{
C. Braban ${ }^{1}$ \& P. Charon ${ }^{2}$ \\ ${ }^{1}$ Siemens Transportation Systems, France \\ ${ }^{2}$ RATP, Paris Urban Transport Operator, France
}

\begin{abstract}
In November 2005, RATP - Paris Urban Transport Operator - awarded Siemens a contract to upgrade the oldest line of the Paris metro to driverless operation (with no driver onboard). This paper discusses RATP's motivations and focuses on the technical challenges for upgrading the ATC from driver-based to driverless operation. This paper also presents the Communications-Based Train Control solution engineered by Siemens.
\end{abstract}

Keywords: communications-based train control, re-signaling, driverless Automatic Train Control.

\section{Introduction}

Since the beginning of the 2000s, RATP, Paris Urban Transport Operator, has undertaken a vast re-signaling program to modernize the automatic train control (ATC) systems installed on its metro network. The overall program aims to increase safety and transport capacity, and improve passenger comfort.

The OURAGAN re-signaling program, focusing on driver-based train operation (Paris lines 3, 5, 9, 10 and 12) to which Siemens Transportation Systems already contributes is part of an overall modernization scheme. In addition to improving safety, capacity and passenger comfort, it aims to achieve high level of parts interchangeability in the communications-based train control system to insure its easy maintainability.

In conjunction with OURAGAN program, feasibility studies have been conducted by RATP to upgrade the existing lines with strong transport demand to driverless lines, following the example of line 14 which is in revenue service since 1998 and driverless from day one. 
The automation of line 1is achieving two goals: the upgrading the PA BF (speed code type of automatic train control solution engineered by Siemens in the early 1970s) to Communications Based Train Control (CBTC), and secondly conversion to full driverless train operation (with no driver onboard), the success on the line 14 is now widely recognized.

The paper explains the motivations of RATP and focuses on the technical challenges for upgrading an existing driver attended line to a driverless one. This paper also presents the Communications-Based Train Control solution engineered by Siemens.

\section{The stakes of upgrading Line 1 to driverless train operation}

\subsection{The oldest line but also the most loaded}

Opened to the public in 1900, Line 1 extends across Paris from the east to the west, a distance of over $17 \mathrm{~km}$. It is the oldest metro line, and also the most prestigious metro line because its 25 stations serve most of the places of interest in the French capital, as well as numerous business districts and shopping centers.

The annual passenger traffic on Line 1 is, at 207 million passengers, the highest in the whole metro network, making it the most heavily used line of the Paris metro. One of the reasons for the high ridership lies in the line's the strategic layout, serving 16 of the most crowded stations, as well as 5 out of 15 major multimodal nodes of the network.

A fleet of 52 MP89 trains operate daily, with a headway of 105 seconds. 23,500 passengers are transported per hour and per direction. Thanks to a commercial speed of $27 \mathrm{~km} / \mathrm{h}$, the estimated travel time from Château de Vincennes (in the east) and la Défense (in the west) is only 35 minutes.

The characteristics of line 1 mentioned above lead to a high request for adaptability of the transport offer, which is hardly reachable with a driver operated line.

Another factor in support of the choice of fully automating line 1 is the necessity of renewing the current equipment before 2010. This particularly concerns signaling, OCC, ATC and interlocking equipment which are 4 major parts of the automation system. Indeed, the current signaling equipment needed to operate the line consists of:

- The Operation Control Center, which was installed in Boulevard Bourdon in Paris, in 1967 and upgraded in 1981.

- The wayside signaling used for safe train separation, which was installed in 1956.

- The PA BF (Pilotage Automatique Basse Fréquence), the speed code solution engineered by Siemens, used for controlling train movement.

The changeover to driverless train operation will increase transport capacity thanks to a time headway reduction from 105 to 85 seconds. The speed of driver-attended trains is currently limited to $70 \mathrm{~km} / \mathrm{h}$. With driverless trains, the 
maximum speed rises to $80 \mathrm{~km} / \mathrm{h}$, enabling the commercial speed to be increased.

The increasing proportion of delays due to passengers also contributes to the choice of line 1 automation. $72 \%$ of the delays are due to passengers and among them $69 \%$ can be controlled by using platform screen doors (e.g. serious accidents, alarm signal, passenger on tracks, objects on tracks).

Moreover, from an economical point of view, line 1 automation will reduce the operating costs between 10 and 15\%, without taking into account the accidents that are avoided doing so.

Finally, the positive feedback of satisfaction from both passengers and operators on line 14 and the above-mentioned elements convinced RATP that line 1 automation is the best solution.

The line 1 automation program consists of different kinds of projects. Most of them obviously deal with technical issues such as the automatic train control system, which is the main subject of this paper, and also the platform screen doors, the rolling stock, the audio-visual equipment. But there is also an organizational project to upgrade the line operation and jobs. As a consequence, this ambitious program, whose objectives and constraints are shared by many transport operators and authorities around the world, requires a complex financial, social and technical process of design and management under the responsibility of RATP. The main milestones of this program are:

- $\quad$ the start of the platform screen doors installation in January 2008

- the commissioning of the new Operational Control Centre in February 2009

- $\quad$ the first operating driverless train in October 2009

- a fully automated line in 2010

\subsection{The CBTC Line 1 upgrade contract}

On November 7, 2005, RATP and Siemens Transportation Systems France signed a 30.8 million euro contract for upgrading Line 1 from driver-attended to driverless train operation, thus consolidating Siemens' leadership in the design of automatic train control (ATC) solutions for the RATP. This new award follows a number of ATC solutions designed for RATP by Siemens:

- Automatic Train Control for driver-attended lines: PA 135 and PA BF installed on 12 of 13 metro lines.

- Automatic Train Control (SACEM) for RER A and B regional Express lines.

- Driverless Communications-Based Train Control for Line 14 and its extensions to Saint Lazare railway station (in revenue service since December 2003) and Olympiades (in revenue service in 2007),

- Communications-Based Train Control for driver-attended lines as part of the OURAGAN re-signaling program: lines 3 and 5, and subsequently lines 9, 10 and 12. 
The upgrading Line 1 to a driverless line presents real technical challenges. The challenges are unique because the switchover from the current operation (using a fleet of trains with drivers under the control of a speed code solution) to driverless operations (using a free-propagation CBTC solution) will have to be carried out without any disruption of passenger service.

The contract comprises the:

- Design and supply of equipment necessary for driverless train operation: wayside equipment and onboard equipment installed on 49 cars.

- Supply of the Data Communication System based on free propagation radio, comprising the wayside backbone network and equipment for wayside-to-train communication.

- The Operation Control Center (OCC), which enables RATP to supervise and manage operations on the line.

The cut-over strategy that enables the switchover from driver-attended train operation to driverless operation consists of three key milestones:

1. Refurbishment of the existing OCC to meet specific requirements of future driverless train operation. The new OCC is then put in service to supervise and manage current driver-attended trains operation.

2. Operation of the first driverless train.

3. Mixed-mode operation: both manual operated and driverless trains run on the line during a interim period. Progressive replacement of driver-operated trains by driverless ones until the entire Line 1 fleet is running in driverless mode.

To achieve these milestones a number of technical requirements must be satisfied:

- The system will have to support mixed-mode operation: During a given time period, driver-operated and driverless trains will share the line during daily operation.

- The installation and testing of the system will have to be performed as transparently as possible with respect to daily passenger service. As a consequence, the only possible interruptions of passenger service have to match the normal daily service interruptions, namely at night during an extremely short three hour time window.

\section{The CBTC solution designed for Paris Line 1}

\subsection{Key assets}

The technical solution designed for Line 1 allows for:

- Simultaneous and safe operation of driver-attended and driverless trains thanks to the tracking of CBTC-equipped and unequipped trains. 
- Unequipped trains, operated by drivers, are tracked using the existing track circuits. Movement authorities are delivered to drivers via the existing wayside signaling equipment.

- Driverless trains are tracked based on the location report issued by onboard equipment and delivered to wayside equipment. Movement authorities are then communicated to the trains by wayside equipment based on an exchange of messages. This is the fundamental principle of Communication-Based Train Control.

- Minimal installation work thanks to a significant reduction of wayside equipment as the result of:

- The use of the free-propagation radio solution engineered by Siemens, adaptable to free ISM band frequencies as well as to narrow proprietary bands. The wayside equipment is installed in stations.

- The decentralized system architecture, with equipment installed in technical rooms located in stations, thus reducing the cabling along the line.

To reduce the number of tests performed during daily operation and to maximize the time available for testing at night, Siemens devised a system qualification strategy, based on:

- The design of a simulation platform allowing the entire system to be tested in the factory.

- Tests performed during daily operation in so-called "shadow mode": this permits the observation and analysis of the behavior of the system installed in its real-life environment over a long-time period without impact on passenger service.

- The implementation of sophisticated migration processes, making it possible to switch from the current mode to driverless operation and back again within the time slots available at night dedicated to testing.

\subsection{Focus on train tracking}

The simultaneous, safe operation of driver-attended and driverless trains relies on tracking system based on virtual blocks combined with the moving block principle. This stems from the technical evolution of tracking systems currently in use on the Meteor Line, on the Canarsie Line, on Barcelona line 9 and on OURAGAN.

For the tracking of unequipped trains, the existing track circuits are used. The track is physically divided into "blocks", which only contain one train at a given time. Entry to each block is protected by a signal light (red/green) that informs the driver whether or not the block is already occupied by a train.

The tracking of CBTC equipped trains does not depend on track circuits. Instead, the track is split into "virtual blocks", which overlay the existing physical blocks. Each train calculates its own location on the line and transmits it to the wayside CBTC equipment. 
The wayside CBTC equipment continuously updates the status (occupied or free) of each virtual block based on:

- The location reported by the onboard CBTC equipment and delivered to the CBTC wayside equipment, using free propagation radio.

- The occupancy status of track circuits.

The wayside CBTC equipment then computes for each train a target point, i.e. a danger point, not to be passed in order to prevent a hazardous situation from occurring. The target corresponds to the first danger point located downstream of a train. It can be a block (a track circuit or a virtual block) occupied by a train, a red signal or the end of a territory under CBTC control.

Using free-propagation, the wayside CBTC equipment delivers the target point to each CBTC equipped train. In the event that the target point corresponds to an occupied virtual block, the target point is set to the last reported position of the train ahead. This is the moving block principle. If the target point corresponds to a track circuit occupied by an unequipped train, the target point is set to the entry to the track circuit.

Equipped trains then computes the ATP (Automatic Train Protection) speed curve (which guarantees that the train will come to a stop before the next danger point) and the ATO (Automatic Train Operation) curve.

In the case of unequipped trains, information about the next danger point is delivered to drivers using the existing wayside signaling. A green light means that it is safe to enter the block as it is not occupied by any train. A red light means that it is forbidden to enter the block as it is occupied by a train.

\subsection{Focus on the free propagation radio solution}

Now in operation on the Canarsie Line in New York City, the free propagation radio product offers outstanding performance in the face of the stringent requirements $\mathrm{CBTC}$ in underground railway applications. Indeed, the challenge taken up by Siemens was to adapt the various technical innovations to the specific needs of underground transit applications by favoring system availability in these difficult environments for the propagation of radio waves.

For driverless train operation, availability of the data communication system is essential to enable the CBTC system to safely replace the driver. The radio solution fulfils the following requirements:

- Robustness to guarantee the very high level of availability required, despite the diversity of the environments: alternating between tunnels and open air sections, interference and masking. The signal modulation is based on Direct Spread Spectrum Sequence (DSSS). The demodulation takes into account the energy carried by the various multi-paths to "rebuild" the signal transmitted.

- High performance with respect to message updating and handover constraints. Wayside-to-train messages can be addressed to all the trains or to a set of trains on the line. Messages are refreshed frequently 
(approximately every $0.5 \mathrm{~s}$ ) to ensure maximum performance of the CBTC system.

- Robust intrusion protection to ensure security. As the identity of the communication is determined, there is no need for standardization at the air gap. No interface at the air gap is a guarantee of protection against intentional or accidental intrusion.

- Proven coexistence with ISM band users: no perturbation from WIFI users, no perturbation to WIFI users.

The robustness of the free-propagation radio system was achieved through the thoroughness with which the radio link budget was drawn up in various environments as well as: TDMA and micro-synchronization, diversity, and geographical organization redundancy. As a consequence, it guarantees unrivalled operational availability for a reduced amount of wayside radio equipment. A total of 55 radio equipment installed on $17 \mathrm{~km}$ of the Canarsie Line.

For Line 1, this feature is of key importance as it contributes to the simplification and minimization of the installation work since the free-propagation radio system does not require any continuous medium on the guideway and wayside radio equipment is strategically located in stations.

\subsection{Simulation platform}

In order to minimize the number of tests performed on the line during revenue service hours, and so reduce the risk of disruption to passenger service, considerable efforts are dedicated to the design of powerful simulation tools.

These tools allow extensive in-factory testing including the functioning test of an item (which can be software, a piece of equipment, a subsystem or the complete system) and evaluation of its performance in an environment closely resembling that on the line. The tools are based on key modules comprising the OCC, the wayside signaling, wayside CBTC equipment, CBTC equipped trains, platform screen doors and free-propagation radio system and their corresponding interfaces. Behavior of the items will be assessed both in nominal and degraded conditions based on predefined scenarios. It is possible to interact with the simulation at any time in order to generate events or create failures, thereby increasing the relevance of the test performed.

\subsection{The migration strategy}

The major challenge when re-signaling lines in revenue service lies in the validation of the system - in the specific case of Line 1, the OCC and the CBTC onboard equipment - without impairing the quality of service offered to passengers during daily operation and compromising safety. A fundamental feature of this solution is therefore the ability to be operated in "shadow mode", whereby the system receives and sends all necessary information as if under CBTC control, but without any actual outputs being activated. 
A second challenge which makes this project unique is the coexistence of:

- Totally different automatic train control technologies - speed code and CBTC,

- Different modes of train operation - manually operated trains and driverless trains.

The migration strategy is organized into three main time periods corresponding to the three key milestones scheduled by RATP.

- Period 1 is concerned with all the installation and testing work requested for operating the line with the new OCC. The main technical difficulty lies in testing and commissioning all the functions available in the "new" OCC, while still ensuring the safety of daily train operations under the control of the "old" OCC. This work is carried out during both daily operations and night-time hours. It involves having the two OCC working in parallel: until the first milestone is reached, the "old" one remains active and the "new" one operates in shadow mode; at the end of this period, the "new" OCC becomes active, but the "old" one remains available for operation if necessary.

- Period 2 focuses on the testing and commissioning of the driverless CBTC solution to enable the first driverless train to operate on the line together with manually operated trains. It should be noted that all the wayside CBTC equipment is installed during the Period 1. The testing covers ATP and ATO functions. The main difficulty is how to in perform all the necessary tests and customizations without interfering with work already done. For this, the system will first be extensively tested on the test track in Valenciennes, France. In addition, a CBTC system installed on a MP 89 train will be tested in shadow mode on Line 1.

- Period 3 is concerned with the progressive introduction into revenue service of the new MP05 rolling stock equipped with the driverless CBTC. Manually operated trains are progressively removed from service. The final functionality of the system is tested, including automatic train regulation and management of the depot.

\section{Conclusion}

The upgrading of Line 1 of the Paris metro from a driver-based to a driverless operation (with no driver onboard) is undoubtedly one of the most important projects RATP is going to realize in the next five years.

After the great success achieved with Meteor Line 14, which demonstrated the undeniable advantages of driverless operation, this project opens up the way to re-signaling and upgrading conventional lines to driverless operation.

Two major stakes are associated with the line 1 project. The first one addresses the unquestionable improvement of the quality of service, resulting from the flexibility of driverless operation. The second one is related to complex political and social issues associated with it. 
Switching over from a conventional line operation to a driverless mode of operation is not a simple matter. It includes major technical challenges, i.e. to install, test and commission the complete system in total transparency to daily operation, so as not to inconvenience passengers or reduce safety in any way.

Since its first implementation on Line 14, the solution proposed by Siemens has benefited from the latest technical innovations implemented on the Canarsie Line of New York City Transit, Line 9 of Barcelona metro and lines associated with the OURAGAN program. It not only makes the most of its key features such as the tracking of both equipped and unequipped trains, the freepropagation radio, the system's ability to operate the system in "shadow mode", but also integrates state-of-the art simulation tools allowing the testing in real-life scenarios without disrupting operation to successfully take up the technical challenges.

As for RATP, the success of Line 1 Automation relies on the skills and the technical control of the selected suppliers as well as on the experience and the expertise of the RATP in the operation control, the project management and the system risks management. 